\title{
A highly embedded protostar in SFO 18: IRAS 05417+0907
}

\author{
Piyali Saha ${ }^{1,2 *}$, Maheswar Gopinathan ${ }^{1}$, Manoj Puravankara ${ }^{3}$, \\ Neha Sharma ${ }^{1,2}$, Archana Soam ${ }^{4}$ \\ ${ }^{1}$ Aryabhatta Research Institute of observational sciencES, Nainital, India \\ ${ }^{2}$ Pt. Ravishankar Shukla University, Raipur, India \\ ${ }^{3}$ Tata Institute of Fundamental Research, Pune, India \\ ${ }^{4}$ Korea Astronomy and Space Science Institute (KASI), Daejeon, South Korea
}

\begin{abstract}
Bright-rimmed clouds, located at the periphery of relatively evolved HII regions, are considered to be the sites of star formation possibly triggered by the implosion caused due to the ionizing radiation from nearby massive stars. SFO 18 is one such region showing a bright-rim on the side facing the O-type star, $\lambda$ Ori. A point source, IRAS $05417+0907$, is detected towards the high density region of the cloud. A molecular outflow has been found to be associated with the source. The outflow is directed towards a Herbig-Haro object, HH 175. From the Spitzer and WISE observations, we show evidence of a physical connection between the molecular outflow, IRAS $05417+0907$ and the HH object. The spectral energy distribution constructed using multi-wavelength data shows that the point source is most likely a highly embedded protostar.
\end{abstract}

\section{Introduction}

IRAS $05417+0907\left(\alpha_{2000}=05 \mathrm{~h} 44 \mathrm{~m} 29.8 \mathrm{~s} ; \delta_{2000}=+09 \mathrm{~d} 08 \mathrm{~m} 54 \mathrm{~s}\right)$ is a luminous young stellar object (YSO) located in the tip part of a bright-rimmed cloud SFO 18, located at the edge of the large HII region, S264, excited by the massive O-type star $\lambda$ Ori (Lada et al. 1981; Sugitani et al. 1991). It is embedded in dense gas and dust typical of a protostellar environment. A highly collimated outflow has been discovered in the $\mathrm{H}_{2}$ line emission associated with the source. The size of the outflow is $\sim 7.14^{\prime}$ or $0.83 \mathrm{pc}$, considering the distance of the cloud to be $400 \mathrm{pc}$ (Murdin \& Penston 1977). The emission lines of $\mathrm{H}_{2}$ molecules may be due to the excitation of parental cloud material by the jet emission from this protostar.

Using the 13.7-m radio telescope at Taeduk Radio Astronomical Observatory (TRAO), we made mapping observations in the ${ }^{12} \mathrm{CO}(\mathrm{J}=1-0)$ line towards SFO 18. A $4 \times 4$ grid with 44 " grid spacing was used in these observations. We conducted a multiwavelength study of IRAS 05417+0907, involving photometric data from the Two Micron All Sky Survey (2MASS), Spitzer, the Wide-field Infrared Survey Explorer (WISE), Herschel, and the Submillimetre Common-User Bolometer Ar-

\footnotetext{
*s.piyali16@gmail.com
} 
ray (SCUBA). We present preliminary results computed from the spectral energy distribution (SED) which reveals that the IRAS source is most likely in its early stage of evolution.

\section{Observations and data reduction}

\subsection{TRAO Data}

We carried out mapping observations of a region of $15^{\prime} \times 30^{\prime}$ around SFO 18 in ${ }^{12} \mathrm{CO}(\mathrm{J}=1-0)$ using the SEcond QUabbin Observatory Imaging Array (SEQUOIA) at TRAO which is a millimeter-wave radio observation facility with a single dish with a diameter of $13.7 \mathrm{~m}$ at the Korea Astronomy and Space Science Institute (KASI) in Daejeon, South Korea. The observed data is kept in the Continuum and Line Analysis Single-dish Software (CLASS) format. Data reduction was done by the CLASS software of the GILDAS package ${ }^{1}$.

\subsection{Archival Data}

We acquired data from 2MASS, WISE and SCUBA using the VizieR tool ${ }^{2}$.

We used archival data in the 3.6, 4.5, 5.8, and $8.0 \mu \mathrm{m}$ wavebands taken with the Infrared Array Camera (IRAC; Fazio et al. 2004) of the space-based Spitzer telescope. This region was also observed with the Multiband Imaging Photometer for Spitzer (MIPS) at $24 \mu \mathrm{m}$. The basic calibrated data (BCD) were acquired from Spitzer Heritage Archive (SHA). Mosaicking in each wavelength was performed using the MOsaicker and Point source EXtractor (MOPEX) package provided by the Spitzer Science Centre (SSC) and photometry was done using APEX multiframe photometry, which is a part of MOPEX.

The HSA (Herschel Science Archive) User Interface (HUI) was used to acquire Herschel data for this source. The Photoconductor Array Camera and Spectrometer (PACS) observed it at 100 and $160 \mu \mathrm{m}$ and the Spectral and Photometric Imaging Receiver (SPIRE) also detected it at 250, 350 and $500 \mu \mathrm{m}$. For the photometric analysis we used level 2 images of SPIRE and level 2.5 images of PACS which are processed images provided by the Herschel Science Archive (HSA) instead of raw level 0 images. Photometry was done using the task annularSkyAperturePhotometry in the Herschel Interactive Processing Environment (HIPE) software (14.1.0 version).

The TANSPEC (TIFR-ARIES Near Infrared Spectrometer), coupled with 3.6-m Devasthal Optical Telescope (DOT) is expected to reach a $5 \sigma$ detection of 22.5 mag objects in the $\mathrm{K}$ band with a one hour exposure. IRAS $05417+0907$ has a J band magnitude of $\sim 18.44 \mathrm{mag}$, which is an upper limit. So we expect to be able to achieve good photometric data of this source using TANSPEC with the 3.6-m DOT.

\section{Results and discussions}

\subsection{The Source Driving The Outflow}

We identify the source detected in the Spitzer data as a Class 0/I protostar, deeply embedded in the tip part of the cloud SFO 18, driving the outflow. The IRAC ([5.8-8.0], [3.6-4.5]) color-color diagram can be used to roughly determine different evolutionary stages of identified YSOs. Fig. 1 shows the IRAC colors of the sources, located within a field of $8.8^{\prime} \times 8.8^{\prime}$ around the protostar. The red dashed

\footnotetext{
${ }^{1}$ http://www.iram.fr/IRAMFR/GILDAS

${ }^{2}$ http: //vizier.u-strasbg.fr/
} 


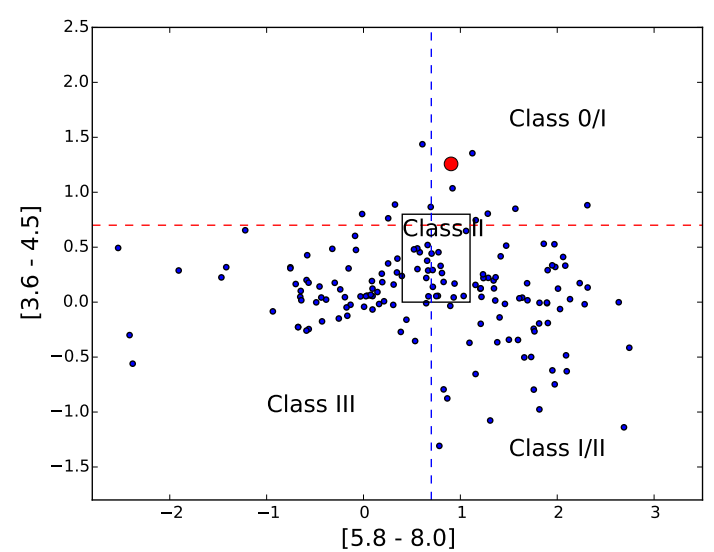

Figure 1: The color-color diagram of IRAS 05417+0907 using the Spitzer 3.6, 4.5, 5.8 and $8.0 \mu \mathrm{m}$ wavebands. IRAS $05417+0907$ is indicated by a red dot.

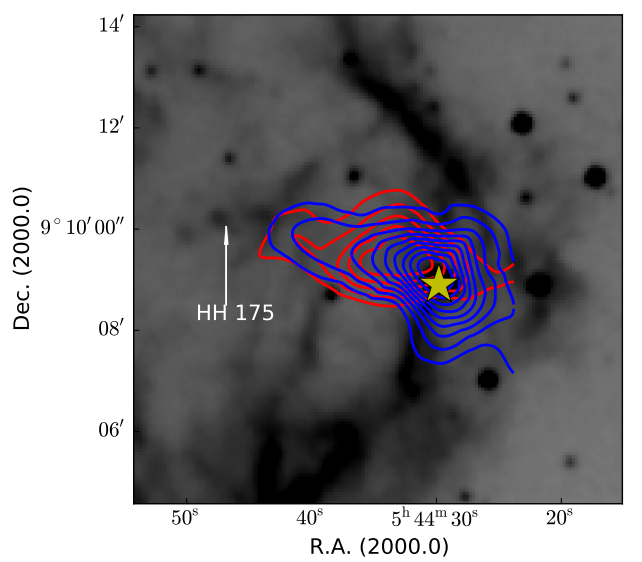

Figure 3: Contour map of ${ }^{12} \mathrm{CO}(\mathrm{J}=1-0)$ line wing integrated intensity in IRAS $05417+0907$ overplotted on the WISE $12 \mu \mathrm{m} 0.5^{\circ} \times 0.5^{\circ}$ image. The redshifted and blueshifted lobes are indicated by red and blue lines, respectively. Blueshifted contours are from 1.2-10.2 $\mathrm{Kkms}^{-1}$ in steps of $1 \mathrm{Kkms}^{-1}$ and redshifted contours range from 1.7-6.6 $\mathrm{Kkms}^{-1}$ in steps of $1 \mathrm{Kkms}^{-1}$. The yellow star marks the IRAS source.

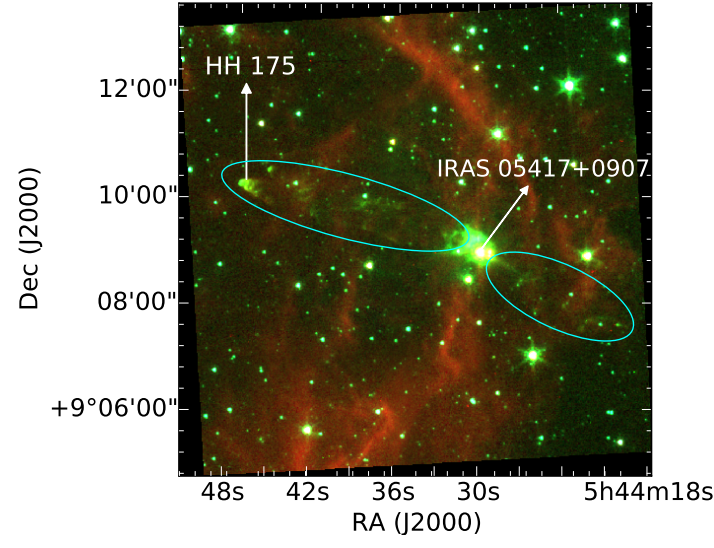

Figure 2: A color-composite image of IRAS $05417+0907$ associated with the outflow. The image is produced from Spitzer-IRAC data adopting blue, green and red for the 3.6, 4.5 and $8.0 \mu \mathrm{m}$ images, respectively.

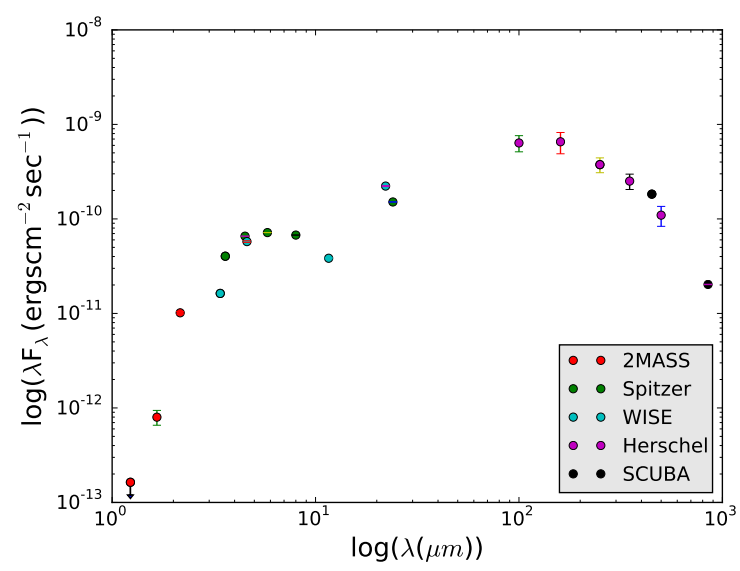

Figure 4: The SED of IRAS 05417+0907 constructed using 2MASS, Spitzer, WISE, Herschel and SCUBA photometric data. 2MASS J band magnitude gives an upper limit, so its corresponding flux is marked as a downward arrow. 
Table 1: Outflow Parameters for the blueshifted (top line) and redshifted (bottom line) lobe associated with IRAS 05417+0907. For each lobe we give the size, the mass M, the momentum P, the energy E, the radial velocity $\mathrm{V}$, the age $\mathrm{t}$, the force $\mathrm{F}$, the mechanical luminosity $\mathrm{L}_{\text {mech }}$ and the mass loss rate $\mathrm{M}_{\text {loss }}$.

\begin{tabular}{|l|ccccccccc|}
\hline Lobe & $\begin{array}{c}\text { size } \\
(\mathrm{pc})\end{array}$ & $\begin{array}{c}\mathrm{M} \\
\left(\mathrm{M}_{\odot}\right)\end{array}$ & $\begin{array}{c}\mathrm{P} \\
\left(\mathrm{M}_{\odot} \mathrm{kms}^{-1}\right)\end{array}$ & $\begin{array}{c}\mathrm{E} \\
(\mathrm{erg})\end{array}$ & $\begin{array}{c}\mathrm{V} \\
\left(\mathrm{kms}^{-1}\right)\end{array}$ & $\begin{array}{c}\mathrm{t} \\
(\mathrm{Myr})\end{array}$ & $\begin{array}{c}\mathrm{F} \\
\left(\mathrm{M}_{\odot} \mathrm{kms}^{-1} \mathrm{yr}^{-1}\right)\end{array}$ & $\begin{array}{c}\mathrm{L}_{\text {mech }} \\
\left(\mathrm{L}_{\odot}\right)\end{array}$ & $\begin{array}{c}\mathrm{M}_{\text {loss }} \\
\left(\mathrm{M}_{\odot} \mathrm{yr}^{-1}\right)\end{array}$ \\
\hline Blue & 0.5 & 0.2 & 0.7 & 0.2 & 3.3 & 0.15 & $0.5 \times 10^{-6}$ & $0.1 \times 10^{-2}$ & $1.3 \times 10^{-7}$ \\
Red & 0.8 & 0.5 & 2.0 & 0.9 & 4.1 & 0.19 & $1.1 \times 10^{-6}$ & $0.4 \times 10^{-2}$ & $2.6 \times 10^{-7}$ \\
\hline
\end{tabular}

horizontal line represents the boundary between Class I/II and Class III (below) and Class 0/I objects (above). The blue dashed vertical line differentiates more evolved Class III sources from younger Class 0/I and Class I/II sources. The rectangle distinguishes the Class II sources from other YSOs (see Megeath et al. 2004). The location of IRAS 05417+0907, indicated by a red dot, suggests that it is a highly embedded protostar.

\subsection{The Outflow}

The Spitzer $4.5 \mu \mathrm{m}$ image reveals a spectacular outflow oriented in northeast-southwest direction associated with IRAS $05417+0907$ which can be seen in the color-composite image using Spitzer 3.6 (blue), 4.5 (green) and 8.0 (red) $\mu \mathrm{m}$ images (Fig. 2). The northeastern part of the outflow ends with a Herbig-Haro object HH 175. The southwestern part is not as much prominent as the other one which may be due to the absence of cloud material in this direction.

The outflow parameters associated with the infrared source have been calculated using ${ }^{12} \mathrm{CO}(\mathrm{J}=1$ 0 ) data. With line center radial velocity at $\mathrm{V}_{L S R}$ of $11.8 \mathrm{kms}^{-1}$, the line wings are integrated from 6-10.5 $\mathrm{kms}^{-1}$ (blue wing) and from 13.5-18 $\mathrm{kms}^{-1}$ (red wing). Contours of line wing emission (redshifted and blueshifted) have been shown in Fig. 3.

From Table 1, we can see the that the outflow has a significantly lower mass $\mathrm{M}$ and mass loss rate $\mathrm{M}_{\text {loss }}$ than those of massive YSOs (Wu et al. 1996). Moreover, low mass sources make up 90\% of outflows associated with $\mathrm{HH}$ objects (Wu et al. 2004) which is the case for IRAS 05417+0907. So, we can consider it to be a young low mass star.

\subsection{The Spectral Energy Distribution:}

The 2MASS 1.23-2.16 $\mu \mathrm{m}$, Spitzer 3.6-8.0 and $24 \mu \mathrm{m}$, WISE 3.4-22.1 $\mu \mathrm{m}$, Herschel PACS 100 and $160 \mu \mathrm{m}$ and SPIRE 250, 350 and $500 \mu \mathrm{m}$, and SCUBA 450 and $850 \mu \mathrm{m}$ data were used to develop the SED of the IRAS source shown in Fig. 4 . The bolometric temperature $\left(\mathrm{T}_{b o l}\right)$ is calculated following the method written in Myers \& Ladd (1993). The bolometric luminosity $\left(\mathrm{L}_{b o l}\right)$ is calculated by integrating over the full SED, and multiplying the result with $4 \pi \mathrm{D}^{2}$, where the $\mathrm{D}$ is the distance of the protostar $(400 \mathrm{pc})$. The submillimeter luminosity $\left(\mathrm{L}_{\text {submm }}\right)$ is calculated by integrating over the SED for $\lambda \geq$ $350 \mu \mathrm{m}$. 


\section{Conclusions}

1. IRAS $05417+0907$ is a luminous YSO which is forming through accretion of the circumstellar disk in the parental cloud SFO 18. A highly collimated outflow has been discovered associated with this protostar in Spitzer $4.5 \mu \mathrm{m}$. The northeastern part of this outflow ends with a Herbig-Haro object HH 175. The southwestern part of the outflow is not as much visible as the northeastern part. The excitation of $\mathrm{H}_{2}$ molecules by the jet might be less efficient here due to the absence of the cloud material.

2. The SED constructed from multi-wavelength data shows that IRAS $05417+0907$ is probably a highly embedded YSO, which is also supported by its location in Spitzer color-color diagram (red dot in Fig. 1).

3. From the SED we estimated that $\mathrm{L}_{b o l}=11.7 \mathrm{~L}_{\odot}, \mathrm{L}_{b o l} / \mathrm{L}_{s u b m m}=16.8$, and $\mathrm{T}_{b o l}=64.2 \mathrm{~K}$. Chen et al. (1995) grouped all YSOs with $\mathrm{T}_{b o l}<70 \mathrm{~K}$ as Class 0 protostars. According to Andre et al. (1993), YSOs with $\mathrm{L}_{b o l} / \mathrm{L}_{\text {submm }}<200$ are Class 0 protostars. So, the Spitzer color-color diagram and physical parameters mentioned above indicate that IRAS 05417+0907 is most likely in its early stage of evolution.

\section{Acknowledgements}

This work made use of 2MASS, WISE and SCUBA data downloaded from SIMBAD database operated by CDS, Strasbourg, France. This is also based on archival data of Spitzer Space Telescope, operated by the Jet Propulsion Laboratory, California Institute of Technology. The Herschel data were obtained from Herschel Science Archive (HSA). ${ }^{12} \mathrm{CO}(\mathrm{J}=1-0)$ molecular line data were acquired using TRAO at KASI, Daejeon, South Korea.

\section{References}

Chen H., Myers P. C., Ladd E. F., Wood D. O. S. 1995, ApJ, 445, 377

Lada C. J., Thronson Jr. H. A., Smith H. A. et al. 1981, ApJL, 251, L91

Myers P. C., Heyer M., Snell R. L., Goldsmith P. F. 1988, ApJ, 324, 907

Murdin P., Penston M. V. 1977, MNRAS, 181, 657

Sugitani K., Fukui Y., Ogura K. 1991, ApJS, 77, 59

Wu Y., Huang M., He J. 1996, A\&AS, 115, 283

Wu Y., Wei Y., Zhao M. et al. 2004, A\&A, 426, 503 